J. Phys. IV France 127 (2005) 43-49

(C) EDP Sciences, Les Ulis

DOI: $10.1051 /$ jp4:2005127008

\title{
Impulsions laser femtosecondes intenses à contraste élevé dans le bleu-vert par amplification directe dans un milieu gazeux
}

\author{
O. Uteza ${ }^{1, *}$, V. Tcheremiskine ${ }^{1,2}$, R. Clady $^{1}$, G. Coustillier ${ }^{1}$, M. Sentis ${ }^{1}$, \\ Ph. Spiga ${ }^{1}$ et L.D. Mikheev ${ }^{2}$ \\ ${ }^{1}$ Laboratoire LP3, UMR 6182, C. 917, 163 avenue de Luminy, 13288 Marseille Cedex 9, \\ France \\ 2 Institut Lebedev, Leninsky Prospekt 53, 119991 Moscou, Russie
}

\begin{abstract}
Résumé. Cet article présente la conception d'une chaîne laser femtoseconde TW visible, associant (i) un oscillateur solide Ti :Sa opéré à $950 \mathrm{~nm}$, (ii) un amplificateur optique à dérive de fréquence permettant l'amplification du signal à $950 \mathrm{~nm}$ au niveau de plusieurs dizaines de $\mathrm{mJ}$, (iii) un système doubleur pour convertir le signal à $475 \mathrm{~nm}$ et l'injecter dans le milieu amplificateur XeF (C-A), (iv) un milieu gazeux excimères $\mathrm{XeF}(\mathrm{C}-\mathrm{A})$ permettant l'amplification directe du signal à $475 \mathrm{~nm}$ à plusieurs centaines de $\mathrm{mJ}$ et ainsi l'obtention d'impulsions TW visibles à contraste élevé.
\end{abstract}

\section{INTRODUCTION}

Depuis plus d'une décennie, des travaux intenses de recherche sont effectués dans le monde entier pour développer des lasers femtosecondes de très forte puissance (domaine TW à PW). Les applications potentielles de ces lasers sont très nombreuses dans les domaines de l'énergétique (allumage thermonucléaire), de l'optique relativiste (accélération de particules, etc.), de la production de sources de rayonnement secondaires (sources $\mathrm{X}$ incohérentes, etc.), du médical ou de la biologie, etc. Cependant, l'un des problèmes les plus importants et non encore résolus dans le domaine des applications des champs intenses est l'obtention d'un contraste temporel ${ }^{1}$ élevé supérieur à $10^{9}$. Lorsque de tels lasers sont focalisés sur une cible, le contraste, actuellement trop faible $\left(\sim 10^{6}\right)$, ne permet pas l'exploration expérimentale précise de domaines de la physique, comme l'optique relativiste ou le comportement de la matière soumis à un rayonnement intense $\left(\mathrm{I}>10^{18} \mathrm{~W} \cdot \mathrm{cm}^{-2}\right)$. L'origine de la faiblesse du contraste est liée à la présence d'Emission Spontanée Amplifiée (ESA), et de distorsions de phase (pré- et post-impulsions, élargissement et déformation du profil temporel) induites lors du procédé d'amplification à dérive de fréquence (ou technique « $\mathrm{CPA} »-$ Chirped Pulse Amplification).

L'objectif de notre projet est d'explorer une nouvelle approche devant permettre la réalisation de chaînes laser femtosecondes intenses et de contraste temporel élevé. L'originalité de conception de ce type de chaînes laser est d'utiliser des milieux solides pour générer les impulsions femtosecondes ( $<100$ fs, faible énergie), puis d'améliorer leur contraste temporel par conversion non-linéaire et, enfin, de recourir à un milieu gazeux pour les amplifier [1,2]. Lorsqu'il s'agit d'augmenter la puissance laser instantanée d'un système laser en bout de chaîne, un milieu gazeux procure un avantage majeur par rapport à un milieu solide, lié à un seuil d'apparition des phénomènes non linéaires beaucoup plus élevé $\left(\mathrm{n}_{2, \mathrm{gaz}} \approx 0.001 \mathrm{n}_{2, \text { solide }}\right.$ typiquement). Cela permet théoriquement l'amplification directe $\mathrm{d}^{\prime}$ 'impulsions ultrabrèves de forte énergie, sans l'emploi de système étireur - compresseur.

*uteza@LP3.univ-mrs.fr; téléphone : 334918292 83; fax : 334918292 89; www.lp3.univ-mrs.fr

1 Rapports d'intensité entre l'impulsion principale et les pieds d'Emission Spontanée Amplifiée ( $\mathrm{I}_{\text {pulse }} / \mathrm{I}_{\mathrm{ESA}}$ ou contraste incohérent) ou les pré-impulsions laser ( $\mathrm{I}_{\text {pulse }} / \mathrm{I}_{\text {pré-post-impulsion }}$ ou contraste cohérent). 
Parmi les milieux gazeux possibles, le laser à excimères $\mathrm{XeF}(\mathrm{C}-\mathrm{A})$ présente des caractéristiques exceptionnelles d'amplification pour le régime femtoseconde [1,2] : une largeur de gain importante $(\sim 70 \mathrm{~nm})$ centrée à $475 \mathrm{~nm}$ dans une bande accessible par conversion non-linéaire (doublage de fréquences) à partir des lasers Titane:Saphir, une énergie de saturation élevée $\left(\mathrm{E}_{\mathrm{sat}}=0,05 \mathrm{~J} . \mathrm{cm}^{-2}\right)$ et une faible section d'émission stimulée $\left(10^{-17} \mathrm{~cm}^{2}\right)$. De telles propriétés permettent, théoriquement, l'amplification d'impulsions ultrabrèves de $\sim 10$ fs et d'atteindre une intensité de $10^{22} \mathrm{~W} . \mathrm{cm}^{-2}$.

Dans cet article, nous présentons la conception de cette chaîne laser, qui, associe (i) un oscillateur solide Titane:Saphir fonctionnant à $950 \mathrm{~nm}$, (ii) un amplificateur paramétrique optique à dérive de fréquence ou « OPCPA »(Optical Parametric Chirped Pulse Amplification) permettant l'amplification du signal à $950 \mathrm{~nm}$ au niveau de $\sim 50 \mathrm{~mJ}$, (iii) un système doubleur pour convertir le signal à $475 \mathrm{~nm}$ et l'injecter dans le milieu amplificateur $\mathrm{XeF}$ (C-A), (iv) un milieu gazeux excimères XeF (C-A) permettant l'amplification du signal à $475 \mathrm{~nm}$ au niveau de plusieurs dizaines à centaines de $\mathrm{mJ}$ et donc l'obtention d'impulsions TW à contraste élevé dans le bleu-vert.

\section{CONCEPTION DE LA CHAINE LASER}

Récemment, nous avons montré que le rayonnement UV-VUV issu d'un plasma généré par une décharge de surface de forte puissance sur un diélectrique est capable d'exciter de grands volumes de milieux gazeux amplificateur laser (ici $\mathrm{XeF}_{2} / \mathrm{N}_{2} / \mathrm{Ar}$ ou $\mathrm{Kr}$ ) [2]. Nous avons également montré, lors d'expériences pilotes en régime femtoseconde, qu'un gain à faible signal $\mathrm{g}_{0} \sim 1,610^{-3} \mathrm{~cm}^{-1}$, homogène spectralement sur une large plage du domaine d'émission de $\operatorname{XeF}(\mathrm{C}-\mathrm{A})(\Delta \lambda \approx 10 \mathrm{~nm}$ autour de la longueur d'onde de centrage) et spatialement sur un volume de plusieurs litres (section droite $\sim 4 \times 18 \mathrm{~cm}^{2}$ ), pouvait être obtenu pendant une durée submicroseconde [2,3]. L'optimisation, actuellement entreprise de la source de pompage (adjonction d'une deuxième source, augmentation de la puissance déposée, utilisation du krypton comme gaz tampon, etc.), doit permettre de quasiment doubler la valeur du gain à petit signal $\left(\mathrm{g}_{0} \sim 2,510^{-3} \mathrm{~cm}^{-1}\right)$.

Les potentialités du milieu gazeux $\mathrm{XeF}(\mathrm{C}-\mathrm{A})$ pour l'amplification femtoseconde peuvent être estimées à partir des équations suivantes qui donnent la densité d'énergie extraite $\mathrm{E}_{\mathrm{ext}}$ (équation d'amplification de Frantz-Nodvik [4]) et l'importance des distorsions de la phase accumulées sur l'ensemble du trajet de l'impulsion dans le milieu actif (intégrale $\mathrm{B}$ [4]) à partir des propriétés du matériau amplificateur pour différentes impulsions incidentes $\left(\mathrm{E}_{\mathrm{in}}, \mathrm{t}_{\mathrm{in}}\right)$ :

$$
E_{\text {ext }}=E_{\text {sat }} \ln \left\{1+\left[\exp \left(\frac{E_{\text {in }}}{E_{\text {sat }}}\right)-1\right] \exp \left(g_{0} l\right)\right\},
$$

$$
B(t)=\frac{2 \pi}{\lambda} \int n_{2} I(t, l) d l
$$

avec $\mathrm{n}_{2}$, l'indice non-linéaire du milieu, I, l'intensité laser et 1 , la longueur de gain. Le volume gazeux excité occupant à peu près l'ensemble de la cellule, la longueur parcourue par le faisceau dans le milieu est très peu différente de cette dernière et nous prendrons ces deux longueurs égales dans les calculs. Nous assumons un milieu actif de longueur de gain 1 égale à $10 \mathrm{~m}$, aisément accessible par un système multipassages ascendant - descendant dans le milieu gazeux XeF [2], et de gain égal successivement à $\mathrm{g}_{0}=1.610^{-3} \mathrm{~cm}^{-1}$ (vérifié expérimentalement) et $\mathrm{g}_{0} \sim 2,510^{-3} \mathrm{~cm}^{-1}$ (valeur après l'optimisation en cours de la source de pompage). Pour tenir compte de la possibilité de régler le système optique pour permettre un parcours plus long dans la cavité ou de mettre en cascade deux systèmes amplificateurs identiques, nous étudions également deux autres cas correspondant à $\mathrm{g}_{0} \times 21=3,2$ et 5 . L'impulsion

\footnotetext{
2 Il est accepté que B doit être maintenu de l'ordre de l'unité pour préserver la phase du faisceau amplifié et, en particulier, éviter ainsi toute distorsion conduisant à des effets non-linéaires destructifs (auto-focalisation, etc.) [4,5]. Dans les calculs, nous prendrons le critère $\mathrm{B}=1$.
} 
incidente est supposée faiblement divergente $(0,1 \mathrm{mrad})$ et sa fluence varie de 1 à $10 \mathrm{~mJ} . \mathrm{cm}^{-2}$. Le milieu amplificateur est divisé en tranches pour décrire le plus fidèlement possible l'amplification de l'impulsion et l'évolution de l'intégrale B. Les pertes du milieu sont négligées et le gain est supposé constant sur l'ensemble du trajet du faisceau. Nous ignorons les effets de repompage et de déplétion du gain lié aux passages de l'impulsion dans les mêmes zones de gain et au développement de l'ESA. Cela conduit à une légère surestimation des résultats énergétiques et de l'intégrale $\mathrm{B}$. L'indice non-linéaire de réfraction $\mathrm{n}_{2}\left(\mathrm{n}_{2} \approx 2.410^{-19} \mathrm{~cm}^{2} . \mathrm{W}^{-1}\right)$ est calculé pour une composition et des pressions $\left(\mathrm{XeF}_{2} / \mathrm{N}_{2} / \mathrm{Ar}\right.$ dans les proportions respectives 1:37:730, à une pression totale de 1 bar) typiques du milieu photolytique $\mathrm{XeF}(\mathrm{C}-\mathrm{A})[3]$.

Les résultats, présentés sur les figures 1 à 3, montrent qu'il est possible d'amplifier une impulsion femtoseconde directement, sans étireur - compresseur, dans le milieu $\mathrm{XeF}(\mathrm{C}-\mathrm{A})$ à des fluences relativement importantes (jusqu'à $\sim 50 \mathrm{~mJ} . \mathrm{cm}^{-2}$ ), avec un gain en puissance de 5 (pour $\mathrm{g}_{0} \times l=1,6$ ), de 10 (pour $\mathrm{g}_{0} \times l=2,5$ ), et de 50 (pour $\mathrm{g}_{0} \times 2 l=5$ ). Il apparaît également qu'il est préférable, à énergies totales extraites égales, d'avoir une impulsion incidente de faible densité d'énergie afin de limiter l'intensité moyenne présente dans l'amplificateur et donc de limiter le développement des aberrations de phase (intégrale B). Le milieu amplificateur gazeux ayant une section droite importante $\left(>>\mathrm{cm}^{2}\right)$, il est possible (et préférable) d'utiliser une impulsion incidente de grand diamètre afin d'utiliser l'ensemble du milieu actif. De plus, afin de ne pas placer des contraintes trop importantes sur la réalisation de l'impulsion incidente à $475 \mathrm{~nm}$, il est désirable de réaliser une impulsion femtoseconde incidente d'énergie modérée (5 à $10 \mathrm{~mJ}$, $475 \mathrm{~nm}$ ) et de diamètre incident dans le milieu amplificateur $\sim 3 \mathrm{~cm}$. La figure 3 montre alors que l'on peut obtenir, sans l'emploi d'un système compresseur coûteux, peu efficace et complexe à mettre en œuvre, une impulsion femtoseconde de $0,6 \mathrm{TW}\left(50 \mathrm{~mJ}-90 \mathrm{fs}\right.$, pour $\left.\mathrm{g}_{0} \times l=2,5 \mathrm{et} \mathrm{E}_{\mathrm{in}}=1,4 \mathrm{~mJ} . \mathrm{cm}^{-2}\right)$ dans le visible et de bonne qualité optique $(\mathrm{B} \leq 1)$. Il est important de mentionner que des impulsions multiterawatt peuvent être obtenues en opérant le milieu XeF à moindre pression (on observe en effet à peu près le même gain à
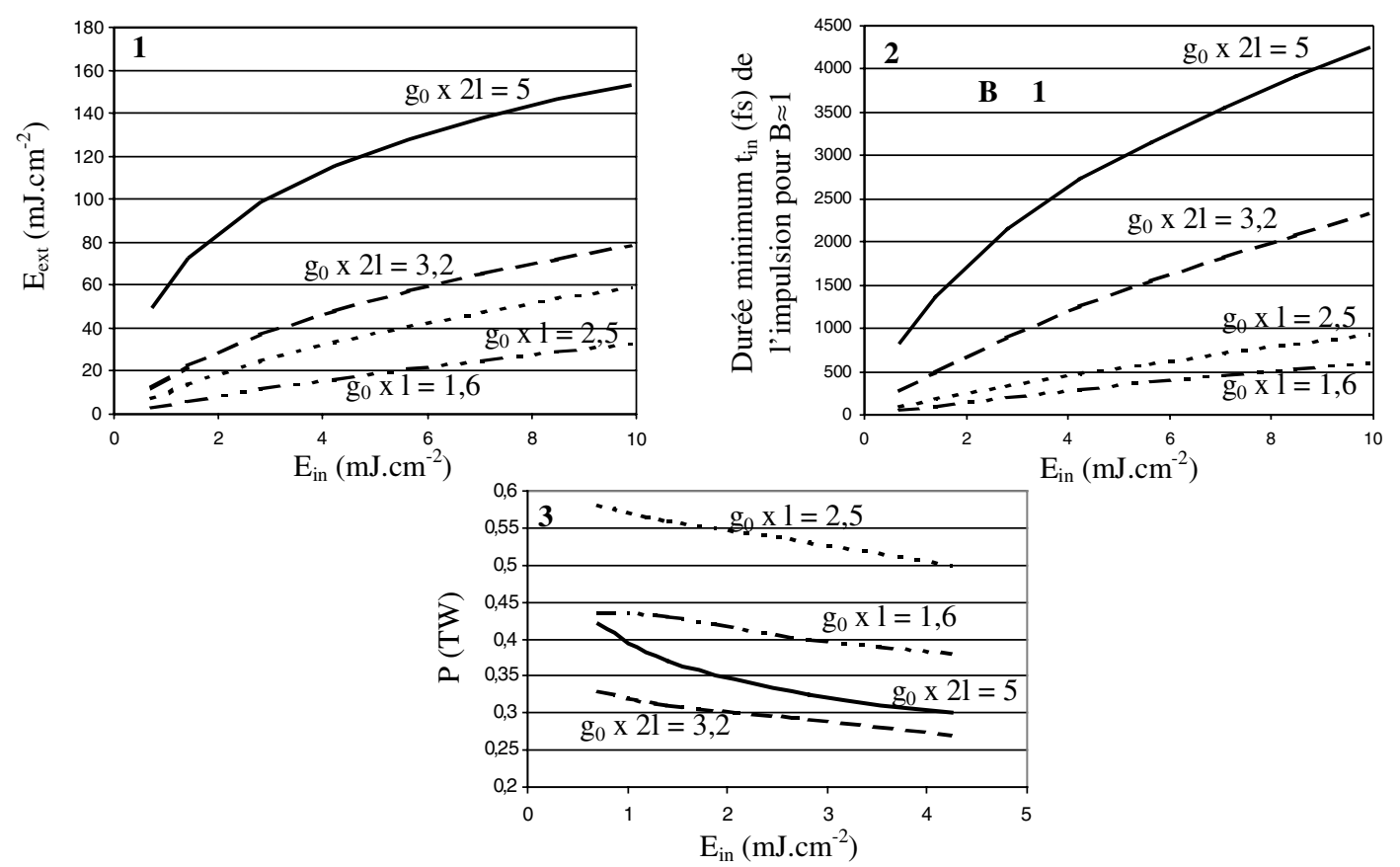

Figures 1-3. Fluence extraite (1), Durée minimum de l'impulsion pour préserver la phase du faisceau $(B \leq 1)(2)$ et Puissance déduite des figs. 1,2 ( $\left.\mathrm{E}_{\mathrm{ext}} \times \mathrm{S} \times \mathrm{t}_{i n, B=1}\right)$ (3) en fonction de la fluence incidente pour un faisceau $\left(\mathrm{S} \approx 7,1 \mathrm{~cm}^{2}\right.$, $\varnothing=3 \mathrm{~cm})$. 
0,3 bar [3]) ce qui permet de réduire $\mathrm{n}_{2}$ et/ou au moyen d'un étage amplificateur de section beaucoup plus grande permettant d'admettre un faisceau incident de plus grand diamètre et donc de réduire de manière importante l'intensité crête du faisceau. Par ailleurs, le gain à petit signal étant faible, le développement de l'ESA est faible (expérimentalement, $\mathrm{I}_{\mathrm{ESA}}<10 \mathrm{~W} \cdot \mathrm{cm}^{-2}$ ), ce qui favorise la conservation d'un contraste élevé, à condition de fournir une impulsion incidente de bon contraste. La géométrie d'amplification hors d'axe et la durée de vie radiative de la transition $\mathrm{XeF}(\mathrm{C}-\mathrm{A})(\sim 100 \mathrm{~ns})$, largement supérieure au temps de transit de l'impulsion utile dans le milieu (environ $30 \mathrm{~ns}$ ), permettront aussi de maintenir un très bon contraste pour l'impulsion amplifiée. En particulier, un contraste optimal sera obtenu pour son front montant si l'on synchronise correctement l'impulsion femtoseconde avec l'excitation du milieu XeF afin que l'amplification de l'impulsion utile domine le développement de l'ESA dans les premiers instants de l'établissement du gain. Compte tenu de la durée de vie radiative de la transition $\mathrm{XeF}(\mathrm{C}-\mathrm{A})$, un synchronisme d'une précision de quelques dizaines de nanosecondes (jtter de l'excitation observé expérimentalement $<50 \mathrm{~ns}$ ) est suffisant.

Par ailleurs, il convient de souligner que l'extraction d'une partie importante de la densité d'énergie stockée $\left(\mathrm{E}_{\text {stockée }} \sim \mathrm{g}_{0} 1 \mathrm{E}_{\mathrm{sat}} \sim 2-5 \mathrm{E}_{\mathrm{sat}}\right.$ ) dans l'étage amplificateur doit se faire en régime picoseconde. Dans ce cas, il faut ensuite prévoir un système de compression après l'étage amplificateur, à prismes si l'intensité est suffisamment faible pour ne pas engendrer de phénomènes d'autofocalisation dans ces derniers ou à réseaux pour les flux les plus intenses. Pour les caractéristiques de l'étage amplificateur XeF ici décrit et pour une impulsion incidente de $10 \mathrm{~mJ}(\varnothing=3 \mathrm{~cm})$, il apparait ainsi possible d'obtenir une impulsion amplifiée d'environ $0,5 \mathrm{~J}$ (pour $\mathrm{g}_{0} \times 2 l=5$ ), soit $\sim 10 \mathrm{TW}$ après recompression dans le régime femtoseconde pour une impulsion initiale de $50 \mathrm{fs}$.

La figure 4 présente l'architecture de la chaîne laser TW, développé par le laboratoire LP3, sur la base des données précédentes. Le produit temps - fréquence est généré par un oscillateur Ti :Sa à modes bloqués par effet Kerr ( $\sim 50 \mathrm{fs}-26 \mathrm{~nm})$. Ce dernier est opéré à $950 \mathrm{~nm}$ pour correspondre après doublage à la bande spectrale d'amplification du milieu XeF. L'impulsion est étirée à une durée de $\sim 1 \mathrm{~ns}$ par un étireur de type Öffner à réseau unique en double passage qui engendre un minimum d'aberrations géométriques [6], puis amplifié par technique OPCPA (Optical Parametric Chirped Pulse Amplification, cf. 3.) [7] au niveau de $\sim 50 \mathrm{~mJ}$. Un compresseur intermédiaire à réseaux de type Treacy [8] (efficacité 50-60\%) permet ensuite d'ajuster la durée de l'impulsion par rapport au régime d'amplification souhaité dans l'étage final XeF (régime femtoseconde : énergie modérée avec amplification directe sans compresseur; régime picoseconde : forte énergie - recompression finale par prismes ou réseaux). L'impulsion est enfin convertie à $475 \mathrm{~nm}$ par doublage de fréquence (efficacité $50-80 \%$ ) ce qui permet de nettoyer l'impulsion et donc d'assurer un très bon contraste à l'impulsion incidente avant son amplification au niveau de 0,5 à $10 \mathrm{TW}$ dans le milieu XeF. Le contraste et une très bonne qualité optique sont conservés grâce aux caractéristiques favorables du milieu XeF $\left(\mathrm{I}_{\mathrm{ESA} \text {,mesuré }}<\right.$ $10 \mathrm{~W} . \mathrm{cm}^{-2}$, matière diluée : faible $\mathrm{n}_{2}$, faible $\mathrm{g}_{0}$, absence d'effets thermiques, pompage optique homogène).

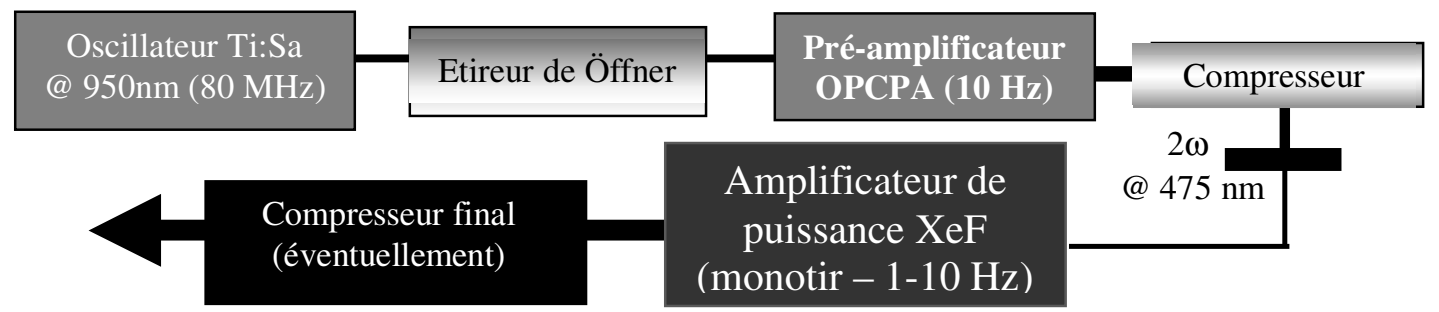

Figure 4. Architecture générale de la chaîne laser. Cellule de Pockels (abaissement de la fréquence) non montrée sur le schéma. 


\section{3. ÉTAGE PRÉ-AMPLIFICATEUR OPCPA}

Cet étage permet de transférer l'énergie de la pompe au faisceau signal utile par interaction paramétrique optique dans un ou plusieurs cristaux. L'impulsion est au préalable étirée dans le domaine nanoseconde pour permettre une amplification à des niveaux énergétiquement importants sans risque d'endommager le matériau non-linéaire et pour pouvoir travailler dans un régime où existent des lasers de pompe efficaces, énergétiques et disponibles commercialement (laser Nd :YaG doublé, etc.).

Mathématiquement, l'interaction paramétrique entre le faisceau pompe (amplitude $\mathrm{A}_{p}$ et pulsation $\left.\omega_{p}\right)$, le faisceau signal étiré $\left(\mathrm{A}_{s}, \omega_{s}\right)$ et l'onde complémentaire ou « idler » $\left(\mathrm{A}_{i}, \omega_{i}\right)$, dans un matériau hôte est décrite par le système d'équations couplées (3) [9]. Les ondes sont supposées planes et d'étendue infinie, d'enveloppe lentement variable et se propageant selon l'axe optique Oz et le milieu non-linéaire est considéré sans pertes et faiblement biréfringent.

$$
\left\{\begin{array}{l}
\frac{d A_{p}}{d z}=i \frac{\omega_{p}}{c n_{p}} d_{e f f} A_{s} A_{i} \exp (i \Delta k z) \\
\frac{d A_{i}}{d z}=i \frac{\omega_{i}}{c n_{i}} d_{e f f} A_{s}^{*} A_{p} \exp (-i \Delta k z) \\
\frac{d A_{s}}{d z}=i \frac{\omega s}{c n_{s}} d_{e f f} A_{i}^{*} A_{p} \exp (-i \Delta k z)
\end{array}\right.
$$

Dans ces équations, c est la vitesse de la lumière, $\mathrm{d}_{e f f}$ le coefficient non-linéaire effectif du cristal, $\mathrm{n}_{p, i, s}$ les indices de réfraction respectifs de la pompe, du complémentaire et du signal, $\Delta \mathrm{k}$ le désaccord de phase, et l'astérique * désigne le complexe conjugué (c.c.). Les ondes complémentaire et signal sont considérées comme la superposition d'ondes planes monochromatiques et la pompe est assumée monochromatique.

Dans notre approche, nous divisons le milieu non-linéaire en tranches suffisamment fines pour considérer, qu'à l'intérieur de chacune d'elles, l'amplitude de la pompe ne varie pas de manière significative. Ceci permet d'une part de simplifier le système d'équations (1) ( $\mathrm{A}_{p} \approx$ cte) et de déterminer une solution où l'amplitude des ondes signal et complémentaire est exprimée en fonction de celles présentes dans la tranche précédente $\left(\mathrm{A}_{i 0}(\mathrm{n})=\mathrm{A}_{i}(\mathrm{n}-1), \mathrm{A}_{i}(0)=0\right.$ à l'entrée du cristal, et $\left.\mathrm{A}_{s 0}(\mathrm{n})=\mathrm{A}_{s}(\mathrm{n}-1)\right)$ (2) et, d'autre part de prendre en compte l'atténuation de la pompe à mesure que son énergie est transférée vers le signal et le complémentaire (conservation de l'énergie appliquée dans chaque tranche).

$$
\left\{\begin{array}{l}
A_{s}(n)=\exp \left(-i \Delta k l_{s} / 2\right)\left[A_{s 0}(n)\left(\operatorname{ch}\left(b l_{s} / 2\right)+i \frac{\Delta k}{b} \operatorname{sh}\left(b l_{s} / 2\right)\right)+i A_{i 0}^{*}(n) \frac{g}{b} \sqrt{\frac{n_{c} \omega_{s}}{n_{s} \omega_{c}}} \operatorname{sh}\left(b l_{s} / 2\right)\right] \\
A_{i}(n)=\exp \left(-i \Delta k l_{s} / 2\right)\left[A_{i 0}(n)\left(\operatorname{ch}\left(b l_{s} / 2\right)+i \frac{\Delta k}{b} \operatorname{sh}\left(b l_{s} / 2\right)\right)+i A_{s 0}^{*}(n) \frac{g}{b} \sqrt{\frac{n_{s} \omega_{i}}{n_{i} \omega_{s}}} \operatorname{sh}\left(b l_{s} / 2\right)\right]
\end{array}\right.
$$

Avec $\mathrm{b}^{2}=\mathrm{g}^{2}-\Delta \mathrm{k}^{2}$ et $\mathrm{g}$, le gain paramétrique par unité de longueur défini par:

$$
g^{2}=\frac{16 \pi^{2} d_{e f f}^{2}}{\lambda_{s} \lambda_{i} n_{s} n_{i}} A_{p} A_{p}^{*}
$$

Une simulation théorique a ensuite été réalisée afin de déterminer un schéma OPCPA permettant d'atteindre les objectifs fixés (énergie maximale - impulsion recompressible). Elle prend en compte la mise en cascade de un ou plusieurs cristaux, la déplétion de la pompe, l'allure temporelle gaussienne et spatiale «top-hat » du faisceau pompe (reproduction fidèle du laser de pompe utilisé : Laser $\mathrm{Nd}: \mathrm{YaG}$ injecté monomode à cavité équipée de miroirs à réflectivité supergaussienne, $450 \mathrm{~mJ} @ 532 \mathrm{~nm}, 8 \mathrm{~ns}$, $10 \mathrm{~Hz}$ ), l'étirement (chirp) de l'impulsion signal (1 ns, enveloppe spectrale gaussienne, $1 \mathrm{~nJ}$ ) et les éventuels décalages temporels entre les impulsions pompe et signal entre chaque cristal. La différence de vitesse de groupe et les effets liés à la dispersion de vitesse de groupe («walk-off » temporel) sont négligés du fait de la durée nanoseconde des impulsions. Les tailles des faisceaux interagissant sont 
prises suffisamment grandes pour minimiser le problème du recouvrement spatial («walk-off » spatial) partiel des impulsions pour les dimensions de cristaux considérés.

Lors d'une récente étude [3], nous avons déterminé que le BBO et un accord de type I (angle d'accord de phase $\theta \approx 23,5^{\circ}$; angle de non-collinéarité $\alpha=1,9^{\circ}$ ) offraient la meilleure configuration pour l'obtention d'un transfert énergétique important entre la pompe à $532 \mathrm{~nm}$ et le signal à $950 \mathrm{~nm}$. Les figures 5 et 6 montrent qu'il est ainsi possible avec cette technique OPCPA d'obtenir une impulsion pré-amplifiée énergétique $(>50 \mathrm{~mJ})$ sans altération significative du spectre et de la phase du signal ce qui permet une recompression aisée du signal et de conserver un très bon contraste avant le doublage de fréquence (figure 6). Ces performances sont obtenues en disposant trois cristaux de BBO en cascade, chacun étant de longueur inférieure à $2 \mathrm{~cm}$ pour minimiser les problèmes de walk-off spatial. La non-prise en compte du walk-off spatial conduit à une surestimation de l'énergie transférée de la pompe au signal. Ce phénomène sera intégré prochainement à la simulation pour obtenir des résultats plus proches de la réalité.
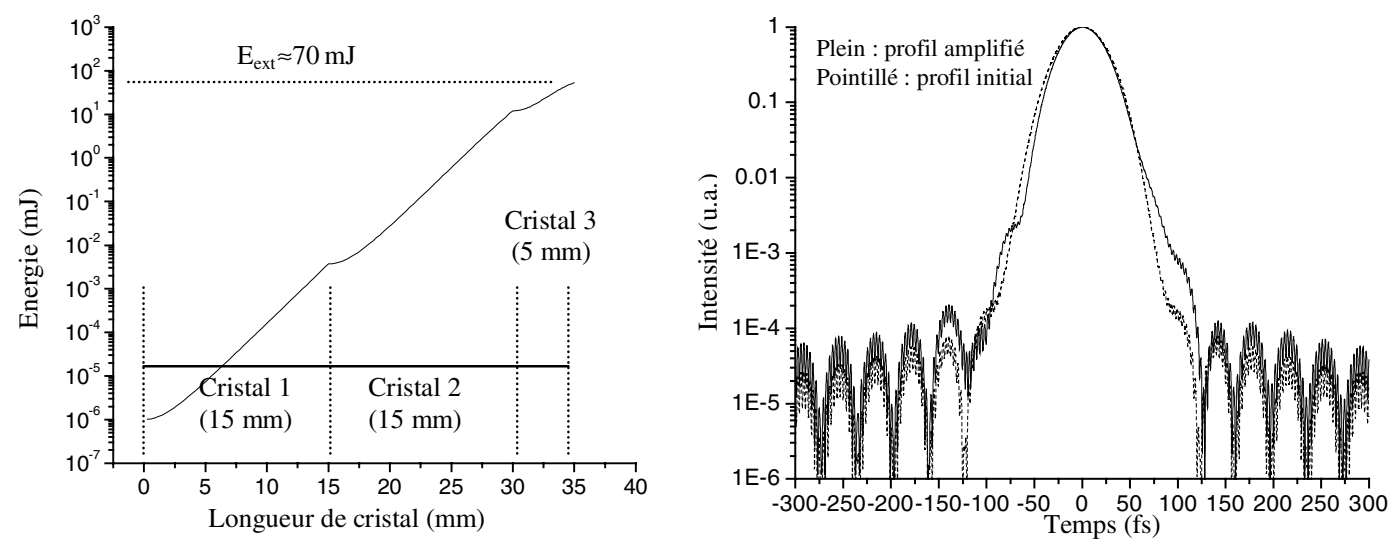

Figures 5 et 6. Energie du signal amplifié en fonction de la longueur de cristal (5, gauche). Reconstruction du profil temporel recompressé après OPCPA pour une impulsion initiale de $1 \mathrm{~nJ}$ - $50 \mathrm{fs} @ 950 \mathrm{~nm}$ (coupure spectrale : 80 $\mathrm{nm}$, phase initiale constante nulle) à partir du spectre et de la phase résiduelle issus de l'OPCPA (6, droite).

\section{CONCLUSIONS}

Cet article présente la conception d'une chaîne laser femtoseconde TW émettant dans le visible (475 nm) et de contraste élevé à partir du milieu amplificateur final gazeux $\mathrm{XeF}(\mathrm{C}-\mathrm{A})$, présentant l'avantage de pouvoir amplifier directement (sans étireur - compresseur) et avec une bonne qualité optique $(\mathrm{B} \leq 1)$ des impulsions femtosecondes. Cette solution hybride, qui associe milieux solides (génération du spectre femtoseconde et pré-amplification OPCPA) et gazeux (amplification) favorise l'obtention d'un contraste très élevé $\left(\mathrm{C}_{\text {théorique }}>10^{11}, \mathrm{I}_{\mathrm{ESA} \text {,mesuré }}<10 \mathrm{~W} \cdot \mathrm{cm}^{-2}\right)$. L'impulsion utile, avant amplification, est en effet obtenue par conversion non-linéaire et acquiert ainsi un contraste avant amplification très élevé. Par la suite, les propriétés du milieu XeF (matière diluée : faible $\mathrm{n}_{2}$, faible gain à petit signal, absence d'effets thermiques, pompage optique homogène, $\mathrm{I}_{\mathrm{ESA} \text {,mesuré }}<10 \mathrm{~W} . \mathrm{cm}^{-2}$ ) doivent permettre de conserver un très bon contraste et une très bonne qualité optique. Une telle chaîne pourrait ainsi constituer une solution à l'un des problème majeurs (faiblesse du contraste) pour l'utilisation des lasers ultrabrefs de puissance.

Cette chaîne laser, de contraste et brillance très élevés dans le bleu-vert, est complémentaire en termes de longueur d'onde des chaînes laser TW solides (IR) et a potentiellement un grand intérêt pour de nombreuses applications dans le domaine de la physique des champs intenses (allumage de réactions nucléaires, génération de faisceaux d'électrons femtosecondes intenses et de rayons X femtosecondes par plasma assisté par laser, accélération de particules). Ainsi, par exemple, une longueur d'onde courte 
pourra améliorer la brillance des sources $\mathrm{X}$ secondaires créées par plasma assisté par laser grâce à l'accroissement du rendement de conversion énergétique (lié à une meilleure absorption du rayonnement laser) et à la ponctualité de la source (liée à une focalisation sur un diamètre plus petit). Dans le même temps, un contraste élevé favorise l'obtention d'un bon rendement énergétique et d'une répartition spectrale énergétique étroite et ciblée (meilleur contrôle). En effet, il n'existe pas dans ce cas de déperdition énergétique lié à la création d'un pré-plasma à la surface de la cible et à son interaction avec le faisceau laser. Par ailleurs, le domaine spectral (bleu-vert) de cette chaîne laser correspond à une zone de transparence de l'eau, ce qui est particulièrement intéressant pour la femtochimie (dynamique réactionnelle en milieu aqueux), la biologie et la médecine.

\section{Remerciements}

Les auteurs remercient la Communauté Européenne dans le cadre du Programme Européen R\&D SHARP (n ${ }^{\circ}$ contrat HPRI-CT-2001-50037) et la Région PACA pour leur soutien financier à ces travaux.

\section{Bibliographie}

[1] L.D. Mikheev, Lasers and Particle Beams 10 (3), 473, 1992.

[2] V.I. Tcheremiskine, M.L. Sentis, L.D. Mikheev, Appl. Phys. Lett. 81 (3), 403, 2002.

[3] O. Uteza, V. Tcheremiskine, R. Clady, G. Coustillier, M. Gastaud, S. Gueguen, M. Sentis, Ph. Spiga, L.D. Mikheev, SPIE Proceedings 5448 of the High Power Laser Ablation conference, Taos, USA, Avril 2004, à paraître.

[4] W. Koechner, in Solid-sate laser engineering ( $5^{\text {th }}$ ed.), ed. Springer, Berlin, 1999.

[5] G. Mourou, T. Tajima, S. Bulanov, Optics in the relativistic regime, à paraître.

[6] G. Chériaux, P. Rousseau, F. Salin, J.P. Chambaret, B. Walker, L.F. Dimauro, Opt. Lett. 21 (6), 414, 1996.

[7] I.N. Ross, P. Matousek, M. Towrie, A.J. Langley, J.L. Collier, Opt. Communications 144, 125, 1997.

[8] E. Treacy, IEEE J. of Quantum Electron. 5 (9), 454, 1969.

[9] R.L. Sutherland, in Handbook of nonlinear optics, ed. M. Dekker, New-York, 1996. 